\title{
Space of minimal discs and its compactification
}

\section{Paul Creutz ${ }^{1}$ [D}

Received: 6 August 2019 / Accepted: 15 May 2020 / Published online: 25 May 2020

(c) The Author(s) 2020

\begin{abstract}
We investigate the class of geodesic metric discs satisfying a uniform quadratic isoperimetric inequality and uniform bounds on the length of the boundary circle. We show that the closure of this class as a subset of Gromov-Hausdorff space is intimately related to the class of geodesic metric disc retracts satisfying comparable bounds. This kind of discs naturally come up in the context of the solution of Plateau's problem in metric spaces by Lytchak and Wenger as generalizations of minimal surfaces.
\end{abstract}

\section{Introduction}

\subsection{Main result}

Since Gromov stated his precompactness criterion in [7] the study of compact and precompact subsets of Gromov-Hausdorff space of metric spaces became a vivid field. Given a precompact class of metric spaces it is a natural but usually very hard question to determine its closure with respect to Gromov-Hausdorff distance. Various partial results have been obtained in the situation where one considers classes of Riemannian manifolds satisfying uniform bounds on parameters such as curvature, volume and diameter. See for example [6,8], [2] and [3] for some of the most important ones. In this article we investigate the class of geodesic metric discs satisfying a uniform quadratic isoperimetric inequality and upper bound on the length of the boundary circle. Such discs come up in [15] as a replacement for minimal surfaces in a quite general metric space setting. The main result of this paper shows that the closure of this class is intimately related to the space of geodesic metric disc retracts in the sense of [19] satisfying comparable bounds. One should note that even without further geometric assumptions it should be possible to understand the topology of Gromov-Hausdorff limits of geodesic metric discs. Compare for example the 2-dimensional results in [24], [22] and [8]. However the arising spaces will be topologically more complicated than disc retracts in general.

The author was partially supported by the DFG grant SPP 2026.

\footnotetext{
$\bowtie \quad$ Paul Creutz

pcreutz@math.uni-koeln.de

1 Mathematisches Institut der Universität zu Köln, Weyertal 86-90, 50931 Köln, Germany
} 
We say that a geodesic metric disc $Z$ satisfies a $C$-quadratic isoperimetric inequality if for every Jordan domain $U \subset Z$ one has

$$
\mathcal{H}^{2}(U) \leq C \cdot l(\partial U)^{2} .
$$

We call a metric space $Z$ a disc retract if there exists a closed curve $\gamma: S^{1} \rightarrow Z$ such that the mapping cylinder $Z_{\gamma}$ is a topological disc. We write $l(\partial Z) \leq L$ if there exists such $\gamma$ satisfying $l(\gamma) \leq L$.

Theorem 1.1 Let $C, L \in(0, \infty)$. Denote by $\mathcal{D}(L, C)$ the set of geodesic metric discs $Z$ satisfying a $C$-quadratic isoperimetric inequality and $l(\partial Z) \leq L$. And by $\mathcal{E}(L, C)$ the set of geodesic metric disc retracts satisfying the same two properties.

Then $\mathcal{E}(L, C)$ is compact and

$$
\overline{\mathcal{D}(L, C)} \subset \mathcal{E}(L, C) \subset \overline{\mathcal{D}\left(L, C+(2 \pi)^{-1}\right)} .
$$

where $\overline{\mathcal{D}(L, C)}$ denotes the closure of $\mathcal{D}(L, C)$ with respect to Gromov-Hausdorff distance. It has been shown in [16] that a proper geodesic metric space $X$ satisfies a $(4 \pi)^{-1}$-quadratic isoperimetric inequality iff it is a CAT(0)-space. In this light our Theorem 1.1 covers as a special case the compactness lemma in [19] which states that the class of CAT(0) disc retracts $Z$ satisfying $l(\partial Z) \leq L$ is compact.

Note that a proper geodesic metric space which satisfies a $C$-quadratic isoperimetric inequality where $C<(4 \pi)^{-1}$ is a metric tree and hence not a metric disc, see [18,23]. So at least in general one cannot have $\overline{\mathcal{D}(L, C)}=\mathcal{E}(L, C)$.

\subsection{Byproducts}

The classical problem of Plateau formulated in the metric space setting asks whether for a given Jordan curve $\Gamma$ in a metric space $X$ there exists a Sobolev disc $u \in W^{1,2}(D, X)$ of least area spanning $\Gamma$. Lytchak and Wenger solved Plateau's problem for proper metric spaces in [13]. Similar to the intrinsic metric on an immersed submanifold one may associate to a solution $u$ of Plateau's problem a geodesic metric space $Z_{u}$. If $X$ satisfies a $C$-quadratic isoperimetric inequality, then $Z_{u}$ is a geodesic metric disc satisfying a $C$-quadratic isoperimetric inequality and $l\left(\partial Z_{u}\right)=l(\Gamma)$, see [15]. Here we say that $X$ satisfies a $C$-quadratic isoperimetric inequality if every closed Lipschitz curve $\gamma: S^{1} \rightarrow X$ admits a Sobolev disc $v \in W^{1,2}(D, X)$ spanning $\gamma$ such that

$$
\operatorname{Area}(v) \leq C \cdot l(\gamma)^{2}
$$

This definition is in consistent with (1) if $X=Z$ is a geodesic metric disc, see [5,17]. In Sect. 2.3 we prove the following.

Theorem 1.2 Let $Z \in \mathcal{D}(L, C)$ and $u \in W^{1,2}(D, Z)$ be a solution of Plateau's problem for the curve $\partial Z$ in $Z$. Then $Z_{u}$ is isometric to $Z$.

So it is natural to think of elements of $\mathcal{D}(L, C)$ as intrinsic minimal discs. In particular Theorem 1.2 allows to apply the structural results of [15] to elements of $\mathcal{D}(L, C)$. One consequence of this is that $\mathcal{D}(L, C)$ is precompact in Gromov-Hausdorff space.

If additionally one imposes a uniform condition avoiding degeneration of the boundary curve, then one only needs to adjoin the trivial metric space $\{*\}$ to compactify. A Jordan curve $\Gamma$ in a metric space $X$ is said to be $\lambda$-chord-arc where $\lambda \geq 1$ if for all $x, y \in \Gamma$ the length of the shorter of the two arcs connecting $x$ and $y$ in $\Gamma$ is bounded above by $\lambda \cdot d(x, y)$. 
Theorem 1.3 Let $C, L \in(0, \infty), \lambda \in[1, \infty)$ and $\mathcal{D}^{\lambda}(L, C)$ be the set of those $Z \in \mathcal{D}(L, C)$ such that $\partial Z$ is $\lambda$-chord-arc. Then $\mathcal{D}^{\lambda}(L, C) \cup\{*\}$ is compact.

The proof is based on a result from [24] which shows that under certain uniform local contractibility conditions Hausdorff limits of discs are again discs.

In Sect. 3 we discuss how to associate to a disc retract $Z \in \mathcal{E}(L, C)$ a metric on the mapping cylinder $Z_{\gamma}$ such that $Z_{\gamma} \in \mathcal{D}^{1}(L, C+1)$. This general construction might be of independent interest and is also applied in [5]. Using this observation it is not hard to derive Theorem 1.1 from Theorem 1.3.

We call a monotone increasing function $\rho:\left(0, R_{0}\right) \rightarrow \mathbb{R}$ a contractibilty function if $\lim _{r \rightarrow 0} \rho(r)=0$. We say that a metric space $X$ is in LGC $(\rho)$ if for every $x \in X$ and $r \in\left(0, R_{0}\right)$ the ball $B(x, r)$ is contractible inside $B(x, \rho(r))$. Using Whyburn's result in [24] instead of Theorem 1.3 and the same construction we also obtain.

Theorem 1.4 Let $\rho$ be a contractibility function, $L \in(0, \infty)$ and $\left(Z_{n}\right)$ be a sequence of disc retracts such that $Z_{n} \in \operatorname{LGC}(\rho)$ and $l\left(\partial Z_{n}\right) \leq L$. If $\left(Z_{n}\right)$ GH-converges to a compact metric space $Z$, then $Z$ is a disc retract and $l(\partial Z) \leq L$.

\subsection{Local versions}

Let $C \in(0, \infty)$ and $l_{0} \in(0, \infty]$. A metric space $X$ is said to satisfy a $\left(C, l_{0}\right)$-(local) quadratic isoperimetric inequality if for every closed Lipschitz curve $\gamma: S^{1} \rightarrow X$ such that $l(\gamma)<l_{0}$ there exists a disc $u \in W^{1,2}(D, X)$ such that $\operatorname{tr}(u)=\gamma$ and $\operatorname{Area}(u) \leq C \cdot l(\gamma)^{2}$. Easy examples show that the class of geodesic metric discs $Z$ satisfying a $\left(C, l_{0}\right)$-quadratic isoperimetric inequality and $l(\partial Z) \leq L$ is not precompact in Gromov-Hausdorff space. Think for example of cylinders $S^{1} \times[0, R]$ with a spherical cap attached at one end. However this may be fixed by bounding also the two dimensional Hausdorff measure of $Z$.

Let $\mathcal{D}\left(L, A, C, l_{0}\right)$ be the class of geodesic metric discs $Z$ satisfying a $\left(C, l_{0}\right)$-quadratic isoperimetric inequality, $l(\partial Z) \leq L$ and $\mathcal{H}^{2}(Z) \leq A$. Let $\mathcal{E}\left(L, A, C, l_{0}\right)$ be the class of geodesic metric disc retracts satisfying the same bounds. Then we prove that $\mathcal{D}\left(L, A, C, l_{0}\right)$ is precompact and adequately adjusted variants of Theorems. 1.1, 1.2 and 1.3 hold for this class replacing $\mathcal{E}(L, C)$ by $\mathcal{E}\left(L, A, C, l_{0}\right)$ and alike. We will only prove the more general versions below which cover the ones stated above for the special case $l_{0}=\infty$ and $A=C \cdot L^{2}$.

\section{Plateau's problem and intrinsic minimal discs}

\subsection{Reminder: Metric space valued Sobolev maps}

We give a short reminder on metric space valued Sobolev maps. For more details see for example [11,13] and [21].

Let $D:=\left\{v \in \mathbb{R}^{2}|| v \mid<1\right\}$ and $S^{1}:=\left\{v \in \mathbb{R}^{2}|| v \mid=1\right\}, X$ a complete separable metric space and $p \in(1, \infty)$. A measurable map $u: D \rightarrow X$ belongs to $L^{p}(D, X)$ if for every 1-Lipschitz map $f: X \rightarrow \mathbb{R}$ the composition $f \circ u$ belongs to $L^{p}(D)$. A map $u \in L^{p}(D, X)$ belongs to $W^{1, p}(D, X)$ if for every 1-Lipschitz map $f: X \rightarrow \mathbb{R}$ the composition $f \circ u$ lies in the classical Sobolev space $W^{1, p}(D)$ and the Reshetnyak p-energy of $u$

$$
E_{+}^{p}(u):=\inf \left\{\|g\|_{L^{p}(D)}^{p}\left|g \in L^{p}(D): \forall f \in \operatorname{Lip}_{1}(X, \mathbb{R}):\right| \nabla(f \circ u) \mid \leq g \text { a.e. }\right\}
$$

is finite. 
We say that a map $u: D \rightarrow X$ satisfies (Lusin's) property $(N)$ if for every $N \subset D$ with $\mathcal{L}^{2}(N)=0$ one has $\mathcal{H}^{2}(u(N))=0$. For $p>2$ the following Sobolev embedding theorem holds, see for example [13].

Theorem 2.1 Let $p>2$ and $\alpha:=1-\frac{2}{p}$. If $u \in W^{1, p}(D, X)$, then $u$ has a representative $\bar{u} \in C^{\alpha}(\bar{D}, X)$ satisfying Lusin's property $(N)$. Furthermore there is a constant $M=M(p)$ such that

$$
\|\bar{u}\|_{\alpha}:=\sup _{x, y \in \bar{D}} \frac{d(\bar{u}(x), \bar{u}(y))}{|x-y|^{\alpha}} \leq M \cdot\left(E_{+}^{p}(u)\right)^{\frac{1}{p}} .
$$

If $u \in W^{1,2}(D, X)$, then $u$ is approximately metrically differentiable almost everywhere. That is for almost every $x \in D$ there exists a seminorm apmd $x u$ on $\mathbb{R}^{2}$ such that

$$
\operatorname{aplim}_{y \rightarrow x} \frac{d(u(y), u(x))-\left(\operatorname{apmd}_{x} u\right)(y-x)}{|y-x|}=0 .
$$

Define the (Busemann) area $\operatorname{Area}(u) \in[0, \infty)$ of $u \in W^{1,2}(D, X)$ by

$$
\operatorname{Area}(u):=\int_{D} J\left(\operatorname{apmd}_{x} u\right) \mathrm{d} \mathcal{L}^{2}(x) \leq E_{+}^{2}(u)
$$

where for a seminorm $\sigma$ on $\mathbb{R}^{2}$ one sets its (Busemann) Jacobian $J(\sigma)$ to be

$$
J(\sigma):=\frac{\pi}{\mathcal{L}^{2}\left(\left\{v \in \mathbb{R}^{2} \mid \sigma(v) \leq 1\right\}\right)} .
$$

It follows from the definition that for a Möbius transformation $m: D \rightarrow D$ one has Area $(u \circ$ $m)=\operatorname{Area}(u)$ and $E_{+}^{2}(u \circ m)=E_{+}^{2}(u)$. We have the following variant of the area formula which in particular shows, that our definition extends the one used in Riemannian geometry.

Theorem 2.2 ([9]) If $u \in W^{1,2}(D, X)$ satisfies Lusin's property $(N)$, then

$$
\operatorname{Area}(u)=\int_{X} \operatorname{card}\left(u^{-1}(x)\right) d \mathcal{H}^{2}(x) .
$$

For $u \in W^{1,2}(D, X)$ there is a canonical almost everywhere defined trace map $\operatorname{tr}(u) \in$ $L^{2}\left(S^{1}, X\right)$. We write $u \in C^{0}(\bar{D}, X)$ if $u$ extends to a continuous map $\bar{u}: \bar{D} \rightarrow X$. In this case one may simply take $\operatorname{tr}(u)=u_{\mid S^{1}}$.

Let $I=[a, b]$ or $I=S^{1}$. Let $\alpha, \beta: I \rightarrow X$ and $h: I \times[0,1] \rightarrow X$ be Lipschitz maps. We say that $h$ is a Lipschitz homotopy from $\alpha$ to $\beta$ if $h(\cdot, 0)=\alpha$ and $h(\cdot, 1)=\beta$. In this case we write $h: \alpha \rightarrow \beta$. The area of such a Lipschitz homotopy $h$ may be defined similarly as discussed here for Sobolev maps on the disc, see [10]. The following glueing lemma is very useful and will be used repeatedly, see for example Section 2.2 in [12].

Lemma 2.3 Let $\gamma, \eta: S^{1} \rightarrow X$ be closed Lipschitz curves and $h: \gamma \rightarrow \eta$ a Lipschitz homotopy. For $u \in W^{1,2}(D, X)$ such that $\operatorname{tr}(u)=\gamma$, there exists $v \in W^{1,2}(D, X)$ such that $\operatorname{tr}(v)=\eta$ and

$$
\operatorname{Area}(v)=\operatorname{Area}(h)+\operatorname{Area}(u) .
$$

Also the following lower semicontinuouity result will be needed at some point.

Theorem 2.4 ([13,21]) Let $p \geq 2, E \geq 0$ and $\left(u_{j}\right) \subset W^{1, p}(D, X)$. If $E_{+}^{p}\left(u_{j}\right) \leq E$ for all $j$ and $u_{j} \rightarrow{ }_{L^{p}} u$, then $u \in W^{1, p}(D, X)$ and

$$
\operatorname{Area}(u) \leq \liminf \operatorname{Area}\left(u_{j}\right) .
$$




\subsection{Plateau's problem in metric spaces}

Call a continuous map $f: X \rightarrow Y$ between metric spaces $X, Y$ monotone if $f$ is continuous, surjective and $f^{-1}(y)$ is connected for every $y \in Y$.

Let $X$ be a complete metric space and $\Gamma$ a closed Jordan curve in $X$. Let $\Lambda(\Gamma, X)$ be the set of those $u \in W^{1,2}(D, X)$ such that $\operatorname{tr}(u) \in L^{2}\left(S^{1}, X\right)$ has a representative which is a monotone map $S^{1} \rightarrow \Gamma$. Following [15] we call $u \in \Lambda(\Gamma, X)$ a solution of Plateau's problem for $\Gamma$ in $X$ if

$$
\operatorname{Area}(u)=\inf _{v \in \Lambda(\Gamma, X)} \operatorname{Area}(v)
$$

and $u$ minimizes $E_{+}^{2}(u)$ among all $u \in \Lambda(\Gamma, X)$ satisfying (11).

Theorem 2.5 ([13]) Let $X$ be a proper metric space and $\Gamma$ a closed Jordan curve in $X$. If $\Lambda(\Gamma, X) \neq \emptyset$, then there exists a solution $u$ of Plateau's problem for $\Gamma$ in $X$. Every such $u$ satisfies

$$
E_{+}^{2}(u) \leq 2 \cdot \operatorname{Area}(u) .
$$

Here a metric space $X$ is called proper if closed and bounded subsets of $X$ are compact. To gain higher regularity of $u$ one has to impose additional conditions on $X$ and $\Gamma$.

Let $C \in(0, \infty)$ and $l_{0} \in(0, \infty]$. We say that $X$ satisfies a $\left(C, l_{0}\right)$-quadratic isoperimetric inequality if for every Lipschitz curve $\gamma: S^{1} \rightarrow X$ satisfying $l(\gamma)<l_{0}$ there exists $u \in W^{1,2}(D, X)$ such that $\operatorname{tr}(u)=\gamma$ and

$$
\operatorname{Area}(u) \leq C \cdot l(\gamma)^{2} .
$$

If $l_{0}=\infty$, we also simply say that $X$ satisfies a $C$-quadratic isoperimetric inequality. It turns out that a geodesic metric disc $Z$ satisfies a $C$-quadratic isoperimetric inequality iff every Jordan domain $U \subset Z$ satisfies $\mathcal{H}^{2}(U) \leq C \cdot l(\partial U)^{2}$, see [5,17]. For example compact Riemannian and more generally Finsler manifolds, CAT $(\kappa)$-spaces and Banach spaces satisfy some quadratic isoperimetric inequality, see $[4,13]$.

Theorem 2.6 ([12,13]) Let $X$ be a complete metric space satisfying a $\left(C, l_{0}\right)$-quadratic isoperimetric inequality, $\Gamma$ a closed rectifiable Jordan curve in $X$ and $u$ a solution of Plateau's problem for $\Gamma$ in $X$.

Then $u$ has a representative $\bar{u}$ such that $\bar{u} \in C^{0}(\bar{D}, X)$ and $\bar{u}$ satisfies Lusin's property $(N)$. If furthermore $\Gamma$ is $\lambda$-chord-arc and $l(\Gamma)<l_{0}$, then one may find $p=p(C, \lambda)>2$ such that $\bar{u} \in W^{1, p}(D, X)$ and a Möbius transformation $m: D \rightarrow D$ such that

$$
\left(E_{+}^{p}(\bar{u} \circ m)\right)^{\frac{1}{p}} \leq M \cdot\left(E_{+}^{2}(u)\right)^{\frac{1}{2}}
$$

where $M=M(C, \lambda)$.

So if the target space satisfies some quadratic isoperimetric inequality we may always without loss of generality assume that a solution of Plateau's problem $u$ is in $C^{0}(\bar{D}, X)$ and satisfies Lusin's property $(\mathrm{N})$. For disc targets one obtains the following improvement.

Theorem 2.7 ([17]) Let $Z$ be a geodesic metric disc of finite area and boundary length which satisfies a $\left(C, l_{0}\right)$-quadratic isoperimetric inequality. Then there exists a solution $u$ of Plateau's problem for $\partial Z$ in $Z$. Every such $u$ is monotone and furthermore satisfies $\operatorname{Area}(u)=$ $\mathcal{H}^{2}(Z)$. 
This formulation of Theorem 2.7 is contained only implicitly in [17]. So for the convenience of the reader we reproduce the proof.

Proof Every Jordan domain $U \subset Z$ satisfies

$$
\mathcal{H}^{2}(U) \leq C^{\prime} \cdot l(\partial U)^{2}
$$

where $C^{\prime}=\max \left\{C, \frac{\mathcal{H}^{2}(Z)}{l_{0}^{2}}\right\}$. So $Z$ satisfies a $C^{\prime}$-quadratic isoperimetric inequality. As $l(\partial Z)<\infty$ the boundary curve admits a Lipschitz parametrization and hence by the global quadratic isoperimetric inequality one has $\Lambda(\partial Z, Z) \neq \emptyset$. So by Theorem 2.5 there is a solution of Plateau's problem for $\partial Z$ in $Z$.

We prove that $u$ is a solution of Plateau's problem for $\partial Z$ in $Z$ iff

$$
E_{+}^{2}(u)=\inf _{w \in \Lambda(\partial Z, Z)} E_{+}^{2}(w) .
$$

Let $u$ be a solution of Plateau's problem for $\partial Z$ in $Z$ and $v \in \Lambda(\partial Z, Z)$ be such that $E_{+}^{2}(v)=\inf _{w \in \Lambda(\partial Z, Z)} E_{+}^{2}(w)$. By [14, Theorem 4.4] such $v$ exists and may be chosen in $C^{0}(\bar{D}, X)$ and satisfying Lusin's property $(\mathrm{N})$. So by Theorem 2.2

$$
\int_{Z} \operatorname{card}\left(v^{-1}(z)\right) \mathrm{d} \mathcal{H}^{2}(z)=\operatorname{Area}(v) \leq E_{+}^{2}(v)<\infty .
$$

So card $\left(v^{-1}(z)\right)$ is finite $\mathcal{H}^{2}$ almost everywhere in $Z$. But by [17, Theorem 1.2] $v$ is monotone and hence $\operatorname{card}\left(v^{-1}(z)\right)=1$ for $\mathcal{H}^{2}$-almost every $z \in Z$. Using that $u$ satisfies Lusin's property $(\mathrm{N})$ and applying Theorem 2.2 again we obtain

$$
\operatorname{Area}(u) \leq \operatorname{Area}(v)=\mathcal{H}^{2}(Z) \leq \operatorname{Area}(u) .
$$

So $v$ is a solution of Plateau's problem. As $u$ minimizes $E_{+}^{2}$ among all area minimizers vice versa one has that

$$
E_{+}^{2}(u) \leq E_{+}^{2}(v)=\inf _{w \in \Lambda(\partial Z, Z)} E_{+}^{2}(w) \leq E_{+}^{2}(u) .
$$

As minimizers of $E_{+}^{2}$ are monotone and have area equal to $\mathcal{H}^{2}(Z)$ this completes the proof.

\subsection{Intrinsic minimal discs}

In the following let $X$ be a complete metric space and $u \in C^{0}(\bar{D}, X)$. Define a semimetric $d_{u}: \bar{D} \times \bar{D} \rightarrow[0, \infty]$ on $\bar{D}$ by setting

$$
d_{u}(x, y):=\inf \{l(u \circ \eta) \mid \eta:[0,1] \rightarrow \bar{D} \text { continuous, } \eta(0)=x, \eta(1)=y\} .
$$

We call the metric space $Z_{u}$ corresponding to $\left(\bar{D}, d_{u}\right)$ the intrinsic disc associated to $u$. By definition one gets canonical maps $P_{u}: \bar{D} \rightarrow Z_{u}$ and $\hat{u}: Z_{u} \rightarrow X$ such that $\hat{u} \circ P_{u}=u$. For solutions of Plateau's problem $u$ this construction has been studied in detail in [15].

Theorem 2.8 ([15]) Let $X$ be a complete metric space satisfying a $\left(C, l_{0}\right)$-quadratic isoperimetric inequality, $\Gamma$ a rectifiable Jordan curve in $X$ and $u$ a solution of Plateau's problem for $\Gamma$ in $X$. Then we have the following list of properties.

(1) $Z_{u}$ is a geodesic metric disc satisfying $\mathcal{H}^{2}(U) \leq C \cdot l(\partial U)^{2}$ for every Jordan domain $U \subset Z_{u}$ such that $l(\partial U)<l_{0}$. 
(2) $P_{u} \in \Lambda\left(\partial Z_{u}, Z_{u}\right)$ is a solution of Plateau's problem for $\partial Z_{u}$ in $Z_{u}$.

(3) For every curve $\gamma$ in $\bar{D}$ one has $l(u \circ \gamma)=l\left(P_{u} \circ \gamma\right)$ and for every $V \subset \bar{D}$ open $\operatorname{Area}\left(u_{\mid V}\right)=\mathcal{H}^{2}\left(P_{u}(V)\right)$. In particular it holds that $l(\Gamma)=l\left(\partial Z_{u}\right)$ and $\operatorname{Area}(u)=$ $\mathcal{H}^{2}\left(Z_{u}\right)$.

(4) $\hat{u}$ is 1-Lipschitz.

(5) $Z_{u} \in \operatorname{LGC}(\rho)$ where $\rho:\left(0, \frac{l_{0}}{2}\right) \rightarrow \mathbb{R}, \rho(r):=(8 C+1) \cdot r$.

(6) Let $l(\Gamma) \leq L$ and $\operatorname{Area}(u) \leq A$. Then for every $\epsilon>0$ there exists an $\epsilon$-net in $Z_{u}$ with at most $K$ elements where $K=K\left(\epsilon, L, A, C, l_{0}\right)$.

We prove the following more precise version of Theorem 1.2.

Theorem 2.9 Let $Z \in \mathcal{D}\left(L, A, C, l_{0}\right)$. If $u$ is a solution of Plateau's problem for $\partial Z$ in $Z$, then $\hat{u}: Z_{u} \rightarrow Z$ is an isometry.

For $u \in C^{0}(\bar{D}, X)$ two variants of the intrinsic disc metric have been investigated in [19]. Define a semimetric $\left|d_{u}\right|: \bar{D} \times \bar{D} \rightarrow \mathbb{R}$ by setting

$$
\left|d_{u}\right|(x, y):=\inf \{\operatorname{diam}(u(C)) \mid C \subset \bar{D} \text { connected, } x, y \in C\} .
$$

Let $\left|Z_{u}\right|$ be the metric space corresponding to $\left(\bar{D},\left|d_{u}\right|\right)$. Let $\left\langle\left|Z_{u}\right|\right\rangle$ be the metric space obtained by taking the path metric on $\left|Z_{u}\right|$. As $Z_{u}$ is a length space we get the following canonical factorization of $\hat{u}$ by 1-Lipschitz maps

$$
Z_{u} \stackrel{u_{1}}{\rightarrow}\left\langle\left|Z_{u}\right|\right\rangle \stackrel{u_{2}}{\rightarrow}\left|Z_{u}\right| \stackrel{u_{3}}{\rightarrow} X .
$$

Proof The aim is to show that if $X=Z$ is as in Theorem 2.9 then all the maps $u_{i}$ in (22) are isometries and hence so is $\hat{u}$.

$u_{3}$ is an isometry: Let $x, y \in \bar{D}$. Let $\gamma:[0,1] \rightarrow X$ be a geodesic in $Z$ connecting $u(x)$ and $u(y)$. As $\bar{D}$ is compact and $u$ is monotone the set $u^{-1}(\operatorname{im}(\gamma))$ is connected. So

$$
\left|d_{u}\right|(x, y) \leq \operatorname{diam}\left(u\left(u^{-1}(\operatorname{im}(\gamma))\right)\right)=\operatorname{diam}(\operatorname{im}(\gamma))=d(u(x), u(y)) .
$$

As $u_{3}$ is 1-Lipschitz this proves that $u_{3}$ is an isometry.

$u_{2}$ is an isometry: As $\left|Z_{u}\right|$ is isometric to $Z$ also $\left|Z_{u}\right|$ is geodesic. So $u_{2}$ is an isometry.

$u_{1}$ is an isometry: We say that $u$ has no bubbles if for every $z \in Z$ every connected component of $\bar{D} \backslash u^{-1}(z)$ contains a point in $S^{1}$. By Theorem 2.8.2 the map $P_{u}$ is continuous and it thus follows from [19, Proposition 9.3] that if $u$ has no bubbles, then $u_{1}: Z_{u} \rightarrow$ $\left\langle\left|Z_{u}\right|\right\rangle$ is an isometry. So it suffices to prove that $u$ has no bubbles. A proof of this fact is implicitly contained in [15]. In our situation one can also conclude this by the following simple argument.

Assume $U$ was a connected component of $\bar{D} \backslash u^{-1}(z)$ disjoint to $S^{1}$. Then $U$ is open. Define $\tilde{u}: \bar{D} \rightarrow Z$ by setting $\tilde{u}(q):=z$ for $q \in U$ and $\tilde{u}(q):=u(q)$ otherwise. Then $\tilde{u}$ is continuous and restricts to a monotone parametrization of $\partial Z$ on $S^{1}$. So by topological reasons $\tilde{u}$ is surjective. Let $q \in U$ and $w:=u(q)$. Then there is $p \in \bar{D} \backslash \bar{U}$ such that $u(p)=\tilde{u}(p)=w$. As $\partial U \cap u^{-1}(w)=\emptyset$ this proves that $u^{-1}(w)$ is disconnected. A contradiction to $u$ being monotone.

Corollary 2.10 For $L, A, C \in(0, \infty)$ and $l_{0} \in(0, \infty]$ let $\mathcal{D}\left(L, A, C, l_{0}\right)$ be the class of all geodesic metric discs $Z$ satisfying $l(\partial Z) \leq L, \mathcal{H}^{2}(Z) \leq A$ and $a\left(C, l_{0}\right)$-quadratic isoperimetric inequality. Then $\mathcal{D}\left(L, A, C, l_{0}\right)$ is precompact in Gromov-Hausdorff space. 
Proof Let $\epsilon>0$. By Theorems. 2.9 and 2.8.6 there is a universal constant $K$ such that every $Z \in \mathcal{D}\left(L, A, C, l_{0}\right)$ admits an $\epsilon$-net with at most $K$ elements. So by Gromov's compactness criterion, see [7], $\mathcal{D}\left(L, A, C, l_{0}\right)$ is precompact in Gromov-Hausdorff space. Note here that the uniform diameter bound needed in the criterion is automatic for classes consisting of geodesic spaces, see for example [1, Exercise 7.4.14].

\section{Mapping cylinders}

Let $X$ be a compact topological space, $\gamma: S^{1} \rightarrow X$ a closed continuous curve and $I=[0,1]$. The mapping cylinder $X_{\gamma}$ of $\gamma$ is the topological space obtained by glueing $Y:=S^{1} \times I$ to $X$ along the equivalence relation generated by $(t, 0) \sim \gamma(t)$.

Now assume $X$ is a metric space, $\gamma: S^{1} \rightarrow X$ is Lipschitz and $L, R \in(0, \infty)$. Let $d_{Y}$ be the product metric on $Y$ obtained by scaling the standard metric on $I$ by the factor $R$ and the angular distance on $S^{1}$ by the factor $\frac{L}{2 \pi}$. Let $d$ be the maximal semimetric on $X_{\gamma}$ subject to $d(x, y) \leq d_{X}(x, y)$ for $x, y \in X$ and $d(x, y) \leq d_{Y}(x, y)$ for $x, y \in Y$.

Lemma 3.1 If $L \geq 2 \pi \cdot \operatorname{Lip}(\gamma)$, then $d$ is a metric and metrizes the topology of $X_{\gamma}$. We denote $X_{\gamma, L, R}:=\left(X_{\gamma}, d\right)$.

In Lemma 3.1 we measure $\operatorname{Lip}(\gamma)$ with respect to the angular distance on $S^{1}$.

Proof For $x, y \in X_{\gamma}$ set

$$
d^{\prime}(x, y):= \begin{cases}d_{X}(x, y) & ; x, y \in X \\ \min _{t \in S^{1}}\left\{d_{X}(x, \gamma(t))+d_{Y}((t, 0), y)\right\} & ; x \in X, y \in Y \\ \min _{t, s \in S^{1}}\left\{d_{Y}(x, y), d_{Y}(x,(t, 0))+d_{X}(\gamma(t), \gamma(s))+d_{Y}((s, 0), y)\right\} & ; x, y \in Y .\end{cases}
$$

We claim that $d^{\prime}$ is a metric. It is clear that $d^{\prime}$ is well defined, positive definite and symmetric. It remains to check the triangle inequality. Let $x, y, z \in X_{\gamma}$. We treat the case $x, z \in X$ and $y \in Y$ only. All other ones are similar. There exist $t, s \in S^{1}$ such that

$$
\begin{aligned}
d^{\prime}(x, y)+d^{\prime}(y, z) & =d_{X}(x, \gamma(t))+d_{Y}((t, 0), y)+d_{Y}(y,(s, 0))+d_{X}(\gamma(s), z) \\
& \geq d_{X}(x, \gamma(t))+\cdot d_{X}(\gamma(t), \gamma(s))+d_{X}(\gamma(s), z) \\
& \geq d_{X}(x, z)=d^{\prime}(x, z) .
\end{aligned}
$$

So $d^{\prime}$ is a metric and hence $d^{\prime} \leq d$. But due to the triangle inequality also $d \geq d^{\prime}$ and hence $d=d^{\prime}$. As $d$ is a metric and $X \sqcup Y$ is compact the topology generated by $d$ agrees with the quotient topology on $Z$, see for example Exercise 3.1.14 in [1].

Theorem 3.2 In the situation of Lemma 3.1 we have the following list of geometric properties.

1. The inlcusion $X \rightarrow X_{\gamma, L, R}$ is an isometric embedding and $X$ is an R-net in $X_{\gamma, L, R}$. There is a strong deformation retraction $H$ of $X_{\gamma, L, R}$ onto $X$ by 1-Lipschitz maps $H_{t}$ : $X_{\gamma, L, R} \rightarrow X_{\gamma, L, R}$.

2. If $X$ is geodesic, then $X_{\gamma, L, R}$ is geodesic.

3. $\mathcal{H}^{2}\left(X_{\gamma, L, R}\right)=\mathcal{H}^{2}(X)+L R$.

4. If $R \geq L$, then the curve $\gamma_{R}: S^{1} \rightarrow X_{\gamma, L, R}, \gamma_{R}(p):=(p, 1)$ is a 1-chord-arc curve of length $L$.

5. If $X$ is $\operatorname{LGC}(\rho)$ where $\rho:\left(0, R_{0}\right) \rightarrow \mathbb{R}$ is a contractibility function, then $X_{\gamma, L, R}$ is $\operatorname{LGC}(\bar{\rho})$ where $R_{0}^{\prime}:=\min \left\{\frac{R_{0}}{2}, \frac{L}{2}\right\}, \bar{\rho}:\left(0, R_{0}^{\prime}\right) \rightarrow \mathbb{R}, \bar{\rho}(r)=\rho(2 r)+r$. 
6. If $X$ satisfies a $\left(C, l_{0}\right)$-quadratic isoperimetric inequality, then $X_{\gamma, L, R}$ satisfies a $\left(C^{\prime}, l_{0}\right)$ quadratic isoperimetric inequality where $C^{\prime}=C+\max \left\{\frac{1}{2 \pi}, \frac{R}{L}\right\}$.

Proof 1. Define the strong deformation retraction $H$ by setting $H_{t}(p, s):=(p, t s)$ for $(p, s) \in Y$ and $H_{t}(x):=x$ for $x \in X$. The claim follows by the representation (23).

2. This is an immediate consequence of the fact that $X$ and $Y$ are geodesic and the representation (23).

3. By the representation (23) the subset $X_{\gamma, L, R} \backslash X$ is locally isometric to $Y \backslash\left(S^{1} \times\{0\}\right)$. So

$$
\mathcal{H}^{2}\left(X_{\gamma, L, R}\right)=\mathcal{H}^{2}(X)+\mathcal{H}^{2}\left(X_{\gamma, L, R} \backslash X\right)=\mathcal{H}^{2}(X)+\mathcal{H}^{2}(Y)=\mathcal{H}^{2}(X)+L R .
$$

4. For $p, q \in S^{1}$ one has $d\left(\gamma_{R}(p), \gamma_{R}(q)\right)=d_{Y}((p, R),(q, R))$. So $l\left(\gamma_{R}\right)=L$ and $\gamma_{R}$ is 1 -chord-arc.

5. Let $x \in X \subset X_{\gamma, L, R}$ and $r \in\left(0, R_{0}\right)$ be arbitrary. Then for $y \in X_{\gamma, L, R}$ and $t \in[0,1]$ one has

$$
d\left(x, H_{t}(y)\right)=d\left(H_{t}(x), H_{t}(y)\right) \leq d(x, y) .
$$

So for $r \in\left(0, R_{0}\right)$ the homotopy $H$ restricts to a strong deformation retraction of $B^{d}(x, r)$ onto $B^{d_{X}}(x, r)$ and by assumption $B^{d_{X}}(x, r)$ is contractible inside $B^{d_{X}}(x, \rho(r)) \subset$ $B^{d}(x, \rho(r))$.

Now let $x \in X_{\gamma, L, R}$ arbitrary and $0<r<R_{0}^{\prime}$. If $B^{d}(x, r) \cap X=\emptyset$, then $B^{d}(x, r)=B^{d_{Y}}(x, r)$ is homeomorphic to a ball in $\mathbb{R}^{2}$ or the halfplane and hence contractible inside itself. So assume $y \in X \cap B^{d}(x, r)$. Then $B^{d}(x, r) \subset B^{d}(y, 2 r)$. By our previous observation $B^{d}(y, 2 r)$ is contractible inside $B^{d}(y, \rho(2 r))$. But $B^{d}(y, \rho(2 r)) \subset B^{d}(x, \bar{\rho}(r))$. This proofs 11 .

6. Let $\eta: S^{1} \rightarrow X_{\gamma, L, R}$ be a closed Lipschitz curve of length $l(\eta)<l_{0}$ and $D=$ $\max \left\{(2 \pi)^{-1}, L^{-1} R\right\}$.

Claim 3.3 For every $D^{\prime}>D$ there exists a Lipschitz homotopy $h: \eta \rightarrow v$ such that $l(v) \leq l(\eta)$, Area $(h) \leq D^{\prime} \cdot l(\eta)^{2}$ and either $v$ is constant or $\operatorname{im}(v) \subset X$.

Assume the claim holds. By assumption there exists $v \in W^{1,2}\left(D, X_{\gamma, L, R}\right)$ such that $\operatorname{tr}(v)=v$ and $\operatorname{Area}(v) \leq C \cdot l(v)^{2}$. So by Lemma 2.3 and Claim 3.3 for every $D^{\prime}>D$ there exists $u \in W^{1,2}\left(D, X_{\gamma, L, R}\right)$ such that $\operatorname{tr}(u)=\eta$ and

$$
\operatorname{Area}(u)=\operatorname{Area}(h)+\operatorname{Area}(u) \leq D^{\prime} \cdot l(\eta)^{2}+C \cdot l(v)^{2} \leq\left(C+D^{\prime}\right) \cdot l(\eta)^{2} .
$$

So $X_{\gamma, L, R}$ satisfies a $\left(C^{\prime \prime}, l_{0}\right)$-quadratic isoperimetric inequality for every $C^{\prime \prime}>C^{\prime}$. Hence by [5] $X_{\gamma, L, R}$ satisfies a $C^{\prime}$-quadratic isoperimetric inequality.

Proof of Claim 3.3 Let $U:=\eta^{-1}\left(X_{\gamma} \backslash X\right)$. Set $h_{t}^{\eta}(s):=H_{t}(\eta(s))$. Then one has $h^{\eta}: \eta \rightarrow v$ where $l(v) \leq l(\eta), \operatorname{im}(v) \subset X$ and

$$
\operatorname{Area}\left(h^{\eta}\right) \leq R \cdot l\left(\eta_{\mid U}\right) .
$$

We consider three cases.

I. $l(\eta) \geq L$ : In this case setting $h=h^{\eta}$ gives the desired homotopy as

$$
\operatorname{Area}(h) \leq R \cdot l(\eta) \leq \frac{R}{L} \cdot l(\eta)^{2} .
$$


II. $l(\eta)<L$ and $U=S^{1}$ : As $l(\eta)<L$ one may fill $\eta$ as good as a planar curve. So there exists a Lipschitz homotopy $h: \eta \rightarrow v$ such that $v$ is constant and

$$
\operatorname{Area}(h) \leq \frac{1}{4 \pi} l(\eta)^{2} .
$$

III. $l(\eta)<L$ and $U \neq S^{1}$ : Taking $h=h^{\eta}$ would prove Theorem 3.2.12 upon replacing the constant $\frac{1}{2 \pi}$ by 1 . For the finer result one has to homotope each piece of $\eta$ contained in $X_{\gamma} \backslash X$ to $X$ in a more optimal way.

First note that by Reshetnyak's majorization theorem, see [20], for a planar Lipschitz curve $\alpha:[0,1] \rightarrow \mathbb{R}^{2}$ there is a compact convex $C \subset \mathbb{R}^{2}$ and a 1-Lipschitz map $m: C \rightarrow \mathbb{R}^{2}$ such that $m_{\mid \partial C}$ is length preserving and a parametrization of $\alpha \cdot \bar{\beta}$ where $\beta$ is a linear parametrization of the Euclidean segment $[\alpha(0), \alpha(1)]$. Hence there is a Lipschitz homotopy $g: \alpha \rightarrow \beta$ such that

$$
\operatorname{Area}(g) \leq \mathcal{L}^{2}(C) \leq \frac{1}{2} \cdot \frac{1}{4 \pi} \cdot(2 \cdot l(\alpha))^{2}=\frac{1}{2 \pi} \cdot l(\alpha)^{2} .
$$

Decompose $U=\cup_{i=1}^{\infty}\left(v^{i}, w^{i}\right)$ where $\left(v^{i}, w^{i}\right)$ are possibly empty angular intervals. Set $\eta_{1}:=\eta$ and for $i \in \mathbb{N}$ let $k_{i}:=l\left(\eta_{\mid\left(v_{i}, w_{i}\right)}\right)$. Using our observation on planar curves inductively for each $i \in \mathbb{N}$ there exists a Lipschitz homotopy $h^{i}: \eta_{i} \rightarrow \eta_{i+1}$ where $l\left(\eta_{i+1}\right) \leq l\left(\eta_{i}\right)$, $\eta_{i+1}=\eta_{i}$ on $S^{1} \backslash\left(v_{i}, w_{i}\right)$ and $\eta_{i+1}\left(\left(v_{i}, w_{i}\right)\right) \subset X$ such that

$$
\operatorname{Area}\left(h^{i}\right) \leq \frac{1}{2 \pi} \cdot k_{i}^{2}
$$

Now let $\epsilon>0$ be arbitrary and $n \in \mathbb{N}$ such that $\sum_{i=n}^{\infty} k_{i}<\epsilon$. For $h=h_{1} \cdot h_{2} \cdot \ldots \cdot h_{n-1} \cdot h^{\eta_{n}}$ : $\eta \rightarrow v$ one has $l(v) \leq l(\eta), \operatorname{im}(v) \subset X$ and

$$
\operatorname{Area}(h)=\operatorname{Area}\left(h^{\eta_{n}}\right)+\sum_{i=1}^{n-1} \operatorname{Area}\left(h^{i}\right) \leq \epsilon \cdot R+\sum_{i=1}^{n-1} \frac{1}{2 \pi} \cdot k_{i}^{2} \leq \epsilon \cdot R+\frac{1}{2 \pi} \cdot l(\alpha)^{2} .
$$

As $\epsilon>0$ was arbitrary this proves the claim.

\section{Limits of discs}

\subsection{Under chord-arc condition}

The following theorem is a slightly weaker version of (6.4) in [24].

Theorem 4.1 ([24]) Let $\rho:(0, R) \rightarrow \mathbb{R}$ be a contractibility function and $X$ be a compact metric space. Let $Z_{n} \subset X$ be metric discs such that both $Z_{n}$ and $\partial Z_{n}$ are in $\operatorname{LGC}(\rho)$ when endowed with the subspace metric. If $Z_{n} \rightarrow_{H} Z$ and $\partial Z_{n} \rightarrow_{H} S$ where $Z, S \subset X$ are compact and $\rightarrow_{H}$ indicates Hausdorff convergence, then $Z$ is a metric disc and $S=\partial Z$.

We will only use the following corollary of Whyburn's result.

Theorem 4.2 Let $L \in(0, \infty), \lambda \in[1, \infty)$ and $\rho:\left(0, R_{0}\right) \rightarrow \mathbb{R}$ be a contractibility function. Let $\left(Z_{n}\right)$ be a sequence of metric discs such that $Z_{n} \in \operatorname{LGC}(\rho), l\left(\partial Z_{n}\right) \leq L$ and $\partial Z_{n}$ is $\lambda$-chord-arc.

If $Z_{n} \rightarrow_{G H} Z$ for a compact metric space $Z$, then either $Z=\{*\}$ or $Z$ is a metric disc such that $l(\partial Z) \leq L$ and $\partial Z_{n} \rightarrow_{G H} \partial Z$. 
Proof If $l\left(\partial Z_{n}\right) \leq r<R_{0}$ then for $z \in Z_{n}$ one has $\partial Z_{n} \subset B(z, r)$ and there is a homotopy $h$ contracting $B(z, r)$ inside $B(z, \rho(r))$. By topological reasons the image of $h$ must be surjective onto $Z$ and hence $\operatorname{diam}\left(Z_{n}\right) \leq 2 \rho(r)$. So if

$$
\liminf _{n \rightarrow \infty} l\left(\partial Z_{n}\right)=0
$$

then $Z=\{*\}$.

So assume without loss of generality that $l\left(\partial Z_{n}\right) \geq l>0$ for all $n \in \mathbb{N}$. Then $\partial Z_{n} \in$ $\operatorname{LGC}(\hat{\rho})$ where $\hat{R}_{0}:=\frac{l}{2 \lambda}, \hat{\rho}:\left(0, \hat{R}_{0}\right) \rightarrow \mathbb{R}$ and $\hat{\rho}(r)=\lambda \cdot r$. By the proof of Gromov's compactnes criterion in [7] there exists a compact metric space $X$ and isometric embeddings $Z_{n} \hookrightarrow X, Z \hookrightarrow X$ such that $Z_{n} \rightarrow_{H} Z$ with respect to these embeddings. As $l\left(\partial Z_{n}\right)$ is uniformly bounded above, by the Arzela-Ascoli theorem there exists a compact set $S \subset X$ such that $\partial Z_{n} \rightarrow_{H} S$. By Theorem 4.1 one has that $Z$ is a metric disc and $\partial Z=S$. Lower semicontinuity of length implies $l(\partial Z) \leq L$.

Remember that $\mathcal{D}\left(L, A, C, l_{0}\right)$ is the class of geodesic metric discs $Z$ satisfying a $\left(C, l_{0}\right)$ quadratic isoperimetric inequality, $l(\partial Z) \leq L$ and $\mathcal{H}^{2}(Z) \leq A$. Let $\mathcal{D}^{\lambda}\left(L, A, C, l_{0}\right)$ be the set of those $Z \in \mathcal{D}\left(L, A, C, l_{0}\right)$ such that $\partial Z$ is $\lambda$-chord-arc.

Theorem $4.3 \mathcal{D}^{\lambda}\left(L, A, C, l_{0}\right) \cup\{*\}$ is compact.

Proof By Corollary 2.10 the class $\mathcal{D}\left(L, A, C, l_{0}\right)$ is precompact and hence so is $\mathcal{D}^{\lambda}\left(L, A, C, l_{0}\right)$. So it remains to show that $\mathcal{D}^{\lambda}\left(L, A, C, l_{0}\right) \cup\{*\}$ is closed.

Let $Z^{n} \in \mathcal{D}^{\lambda}\left(L, A, C, l_{0}\right)$ and $Z_{n} \rightarrow_{G H} Z$. By Theorem 2.8.5 there is a contractibility function $\rho$ such that $Z^{n} \in \operatorname{LGC}(\rho)$. So Theorem 4.2 implies that either $Z=\{*\}$ or $Z$ is a metric disc such that $l(\partial Z) \leq L$ and $\partial Z_{n} \rightarrow_{G H} \partial Z$. Assume we are in the latter case.

By lower semicontinuouity of length one has that $\partial Z$ is $\lambda$-chord-arc. The conditions of being geodesic and satisfying a $\left(C, l_{0}\right)$-quadratic isoperimetric inequality are both stable under Gromov-Hausdorff limits, see [1] and [5] respectively. Note that the proof of Theorem 1.3 is completed at this point. For the local version it only remains to check $\mathcal{H}^{2}(Z) \leq A$.

As in the proof of Theorem 2.7 all $Z_{n}$ satisfy a $\hat{C}$-quadratic isoperimetric inequality where $\hat{C}=\hat{C}\left(A, C, l_{0}\right)$. So by Theorems. 2.5, 2.6 and 2.7 there exist constants $M \in[0, \infty)$, $p \in(2, \infty)$ depending only on $\hat{C}, \lambda$ and maps

$$
u_{n} \in W^{1, p}\left(D, Z_{n}\right) \cap C^{0}\left(\bar{D}, Z_{n}\right) \cap \Lambda\left(\partial Z_{n}, Z_{n}\right)
$$

such that $\operatorname{Area}\left(u_{n}\right)=\mathcal{H}^{2}\left(Z_{n}\right)$ and

$$
\left(E_{+}^{p}\left(u_{n}\right)\right)^{\frac{1}{p}} \leq M \cdot\left(E_{+}^{2}\left(u_{n}\right)\right)^{\frac{1}{2}} \leq M \sqrt{2 \cdot \operatorname{Area}\left(u_{n}\right)}=M \sqrt{2 \cdot \mathcal{H}^{2}\left(Z_{n}\right)} \leq M \cdot \sqrt{2 A}
$$

for all $n \in \mathbb{N}$. In particular by Theorem 2.1 the maps $u_{n}$ are uniformly Hölder and hence equicontinuous.

As in the proof of Theorem 4.2 we may assume that $Z_{n}$ and $Z$ are subsets of a compact metric space $X$ and $Z_{n} \rightarrow_{H} Z$. Then by Arzela-Ascoli $u_{n}: \bar{D} \rightarrow X$ converges uniformly to a map $u \in C^{0}(\bar{D}, X)$. In particular $u_{n} \rightarrow_{L^{p}} u$ and hence by Theorem 2.4 and (32) $u \in W^{1, p}(D, X)$. So by Theorem $2.1 u$ satisfies Lusin's property (N). As $u_{n}: \bar{D} \rightarrow Z_{n}$ is surjective one has $\operatorname{im}(u)=Z$. So by Theorems. 2.2 and 2.4

$$
\mathcal{H}^{2}(Z) \leq \operatorname{Area}(u) \leq \liminf _{n \rightarrow \infty} \operatorname{Area}\left(u_{n}\right) \leq A
$$

and hence $Z \in \mathcal{D}^{\lambda}\left(L, A, C, l_{0}\right)$. 


\subsection{Without chord-arc condition}

Recall that a metric space $Z$ is called a disc retract if there is a Lipschitz curve $\gamma: S^{1} \rightarrow X$ such that $X_{\gamma}$ is a topological disc. We say that $l(\partial Z) \leq L$ if there exists such $\gamma$ satisfying $l(\gamma) \leq L$. The following Lemma shows that one may always assume $\gamma$ to be parametrized by constant speed.

Lemma 4.4 Let $Z$ be a compact metric space, $\gamma: S^{1} \rightarrow Z$ be a Lipschitz curve and $\bar{\gamma}: S^{1} \rightarrow Z$ the constant speed parametrization of $\gamma$. If $Z_{\gamma}$ is a topological disc, then $Z_{\bar{\gamma}}$ is a topological disc.

Proof Let $M: S^{1} \rightarrow S^{1}$ be monotone and such that $\gamma=\bar{\gamma} \circ M$. Define $P: Z_{\gamma} \rightarrow Z_{\bar{\gamma}}$ by

$$
P(x)= \begin{cases}(M(p), t) & ; x=(p, t) \in S^{1} \times[0,1] \\ x & ; x \in Z\end{cases}
$$

Then $P$ is continuous. Furthermore if $x \in Z$, then $P^{-1}(x)=\{x\}$ and if $p \in S^{1}, t \in(0,1]$, then $P^{-1}((p, t))=I \times\{t\}$ where $I \subset S^{1}$ is a closed interval. So $P$ defines a cell-like map $\bar{D} \cong Z_{\gamma} \rightarrow Z_{\bar{\gamma}}$ such that $P_{\mid \partial Z_{\gamma}}$ is cell-like too. So by Corollary 7.12 in [15] $Z_{\bar{\gamma}}$ is homeomorphic to $\bar{D}$.

Remember that $\mathcal{E}\left(L, A, C, l_{0}\right)$ is the set of geodesic metric disc retracts satisfying a $\left(C, l_{0}\right)$ quadratic isoperimetric inequality, $l(\partial Z) \leq L$ and $\mathcal{H}^{2}(Z) \leq A$.

Theorem 4.5 Let $L, A, C \in(0, \infty)$ and $l_{0} \in(0, \infty]$. Then $\mathcal{E}\left(L, A, C, l_{0}\right)$ is compact and

$$
\overline{\mathcal{D}\left(L, A, C, l_{0}\right)} \subset \mathcal{E}\left(L, A, C, l_{0}\right) \subset \overline{\mathcal{D}\left(L, A^{\prime}, C+(2 \pi)^{-1}, l_{0}\right)}
$$

for every $A^{\prime}>A$.

Proof It is clear that $\mathcal{D}\left(L, A, C, l_{0}\right) \subset \mathcal{E}\left(L, A, C, l_{0}\right)$.

Let $Z \in \mathcal{E}\left(L, A, C, l_{0}\right)$. By Lemma 4.4 there is a constant-speed curve $\gamma: S^{1} \rightarrow X$ such that $l(\gamma) \leq L$ and $Z_{\gamma}$ is a disc. Let $Z^{n}:=Z_{\gamma, L, \frac{1}{n}}$. By Theorem 3.2 for $n>L^{-1}$ one has that $d_{G H}\left(Z, Z^{n}\right) \leq \frac{1}{n}$ and

$$
Z^{n} \in \mathcal{D}\left(L, A+\frac{L}{n}, C+\frac{1}{2 \pi}, l_{0}\right)
$$

So $\mathcal{E}\left(L, A, C, l_{0}\right) \subset \overline{\mathcal{D}\left(L, A^{\prime}, C+(2 \pi)^{-1}, l_{0}\right)}$.

By Corollary $2.10 \mathcal{D}\left(L, A^{\prime}, C+(2 \pi)^{-1}, l_{0}\right)$ is precompact. So the only thing that remains to be checked is that $\mathcal{E}\left(L, A, C, l_{0}\right)$ is closed.

Let $Z^{n} \in \mathcal{E}\left(L, A, C, l_{0}\right)$ and $Z^{n} \rightarrow_{G H} Z$ where $Z$ is a compact metric space. Then as in the proof of Theorem $4.3 Z$ is geodesic and satisfies a $\left(C, l_{0}\right)$-quadratic isoperimetric inequality. Let $\gamma^{n}: S^{1} \rightarrow X$ be constant speed curves such that $l\left(\gamma^{n}\right) \leq L$ and $Z_{\gamma^{n}}^{n}$ is a disc. Again we may assume that all $Z_{n}$ and $Z$ are subsets of a compact metric space $X$ such that $Z^{n} \rightarrow_{H} Z$. As $\gamma^{n}$ are uniformly Lipschitz by the Arzela-Ascoli theorem up to passing to a subsequence there exists a limit curve $\gamma: S^{1} \rightarrow Z$ and $l(\gamma) \leq L$.

Set $W^{n}:=Z_{\gamma^{n}, L, L}^{n}$. Then by Theorem 3.2 one has

$$
W^{n} \in \mathcal{D}^{1}\left(L, A+L^{2}, C+1, l_{0}\right)
$$


and hence by Theorem 4.3 after passing to a subsequence there is

$$
W \in \mathcal{D}^{1}\left(L, A+L^{2}, C+1, l_{0}\right)
$$

such that $W^{n} \rightarrow_{G H} W$. But by the explicit formula (23) in this case $W$ has to be isometric to $Z_{\gamma, L, L}$. So $Z$ is a disc retract and $l(\partial Z) \leq L$. Furthermore by Theorem 3.2.3.

$$
\mathcal{H}^{2}(Z)=\mathcal{H}^{2}(W)-L^{2} \leq A+L^{2}-L^{2}=A .
$$

So $Z \in \mathcal{E}\left(L, A, C, l_{0}\right)$.

Theorem 4.6 Let $\rho$ be a contractibility function, $L \geq 0$ and $Z$ a compact metric space. Let $Z_{n}$ be a sequence of disc retracts such that $l\left(\partial Z_{n}\right) \leq L$ for all $n$ and $Z_{n} \rightarrow_{G H} Z$. Then $Z$ is a disc retract and $l(\partial Z) \leq L$.

Proof Proceed exactly as in the last part of the proof of Theorem 4.5 applying Theorem 3.2.5 instead of Theorem 3.2.6 and Theorem 4.2 instead of Theorem 4.3.

Acknowledgements Open Access funding provided by Projekt DEAL. I would like to thank my PhD advisor Alexander Lytchak for great support in everything. Also I would like to thank the anonymous referee for valuable comments which lead to improvements of the presentation.

Open Access This article is licensed under a Creative Commons Attribution 4.0 International License, which permits use, sharing, adaptation, distribution and reproduction in any medium or format, as long as you give appropriate credit to the original author(s) and the source, provide a link to the Creative Commons licence, and indicate if changes were made. The images or other third party material in this article are included in the article's Creative Commons licence, unless indicated otherwise in a credit line to the material. If material is not included in the article's Creative Commons licence and your intended use is not permitted by statutory regulation or exceeds the permitted use, you will need to obtain permission directly from the copyright holder. To view a copy of this licence, visit http://creativecommons.org/licenses/by/4.0/.

\section{References}

1. Dmitri Burago, Yuri Burago, and Sergei V. Ivanov. A Course in Metric Geometry. Graduate Studies in Mathematics 33. American Mathematical Society,Providence, 2001

2. Burago, Y., Gromov, M.L., Perelman, G.Y.: A.D. Alexandrov spaces with curvature bounded below. Russian Math. Surveys 47(2), 1-58 (1992)

3. Cheeger, Jeff, Colding, Tobias H.: On the structure of spaces with Ricci curvature bounded below. I. J. Differ. Geom. 46(3), 406-480 (1997)

4. Creutz, Paul: Majorization by hemispheres and quadratic isoperimetric constants. Trans. Am. Math. Soc. 373(3), 1577-1596 (2020)

5. Paul Creutz. Plateau's problem for singular curves. Comm. Anal. Geom., to appear

6. Grove, Karsten, Petersen, Peter V., Jyh-Yang, Wu: Geometric finiteness theorems via controlled topology. Invent. Math. 99(1), 205-214 (1990)

7. Gromov, Michail L.: Groups of polynomial growth and expanding maps. Publ. Math. Inst. Hautes Études Sci. 53, 53-78 (1981)

8. Michail L. Gromov: Metric Structures for Riemannian and Non-Riemannian Spaces. Progress in Mathematics, Birkhäuser (1999)

9. Karmanova, Maria B.: Area and co-area formulas for mappings of the Sobolev classes with values in a metric space. Sibirsk. Mat. Zh. 48(4), 778-788 (2007)

10. Kirchheim, Bernd: Rectifiable metric spaces: local structure and regularity of the Hausdorff measure. Proc. Am. Math. Soc. 121(1), 113-123 (1994)

11. Korevaar, Nicholas J., Schoen, Richard M.: Sobolev spaces and harmonic maps for metric space targets. Comm. Anal. Geom. 1(3-4), 561-659 (1993)

12. Lytchak, Alexander, Wenger, Stefan: Regularity of harmonic discs in spaces with quadratic isoperimetric inequality. Calc. Var. Partial Differ. Equ. 55(4), 98 (2016) 
13. Lytchak, Alexander, Wenger, Stefan: Area Minimizing Discs in Metric Spaces. Arch. Ration. Mech. Anal. 223(3), 1123-1182 (2017)

14. Lytchak, Alexander, Wenger, Stefan: Energy and area minimizers in metric spaces. Adv. Calc. Var. 10(4), 407-421 (2017)

15. Lytchak, Alexander, Wenger, Stefan: Intrinsic structure of minimal discs in metric spaces. Geom. Topol. 22(1), 591-644 (2018)

16. Lytchak, Alexander, Wenger, Stefan: Isoperimetric characterization of upper curvature bounds. Acta Math. 221(1), 159-202 (2018)

17. Lytchak, Alexander, Wenger, Stefan: Canonical parameterizations of metric disks. Duke Math. J. 169(4), 761-797 (2020)

18. Alexander Lytchak, Stefan Wenger, and Robert Young. Dehn functions and Hölder extensions in asymptotic cones. J. Reine Angew. Math., to appear

19. Petrunin, Anton, Stadler, Stephan: Metric-minimizing surfaces revisited. Geom. Topol. 23(6), 3111-3139 (2019)

20. Reshetnyak, Yurii G.: Non-expansive maps in a space of curvature no greater than $K$. Sibirsk. Mat. Ž. 9, 918-927 (1968)

21. Reshetnyak, Yurii G.: Sobolev classes of functions with values in a metric space. Sibirsk. Mat. Zh. 38(3), 657-675 (1997)

22. Shioya, Takashi: The limit spaces of two-dimensional manifolds with uniformly bounded integral curvature. Trans. Am. Math. Soc. 351(5), 1765-1801 (1999)

23. Wenger, Stefan: Gromov hyperbolic spaces and the sharp isoperimetric constant. Invent. Math. 171(1), 227-255 (2008)

24. Whyburn, Gordon T.: On sequences and limiting sets. Fund. Math. 25(1), 408-426 (1935)

Publisher's Note Springer Nature remains neutral with regard to jurisdictional claims in published maps and institutional affiliations. 